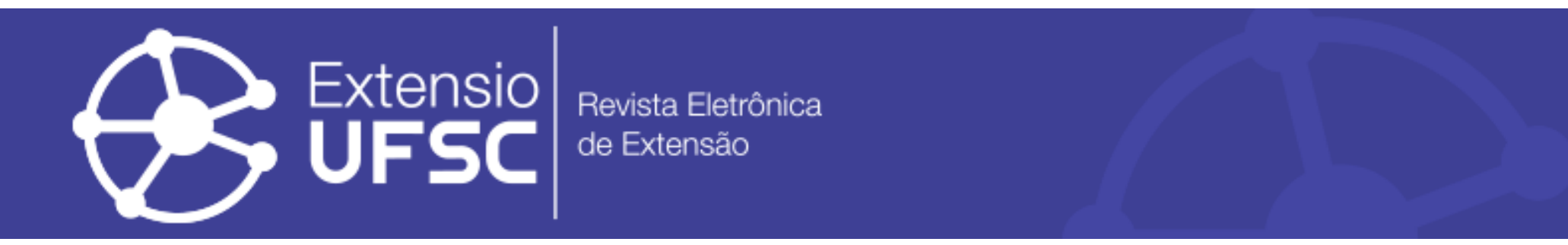

\title{
O IMPACTO DO PROJETO AÇÕES SOCIAIS E DE SAÚDE EM GERONTOLOGIA/UNIVATES (RS) PARA IDOSOS
}

\author{
Bibiana Bünecker Martinez \\ Universidade do Vale do Taquari \\ passgerontologia@univates.br \\ Anna Luiza Ely Thomé \\ Universidade do Vale do Taquari \\ passgerontologia@univates.br
}

\begin{abstract}
Alessandra Cristina Kerkhoff
Universidade do Vale do Taquari passgerontologia@univates.br

Alessandra Brod

Universidade do Vale do Taquari passgerontologia@univates.br
\end{abstract}

Resumo

O século XXI vem sendo significativamente marcado pelo envelhecimento populacional. No Vale do Taquari, o número de habitantes idosos já supera a média nacional, caracterizando $14 \%$ da população total, segundo o Censo de 2010 . O Projeto Ações Sociais e de Saúde em Gerontologia, da Universidade do Vale do Taquari (Univates), trabalha com o público idoso desta região desde 2014. O objetivo deste trabalho foi avaliar o impacto social de tal projeto, junto aos participantes, no ano de 2016. Os idosos responderam sobre suas satisfações em relação ao retorno das avaliações de saúde realizadas, aos temas e informações prestadas durante as oficinas e em relação ao impacto do projeto na vida deles. A média das respostas apontam que os idosos consideraram as ações ótimas.

Palavras- Chave: Envelhecimento. Extensão Universitária. Impacto Social. Idoso.

\section{THE IMPACT OF THE SOCIAL ACTIONS AND HEALTH IN GERONTOLOGY PROJECT/UNIVATES FOR ELDER PARTICIPANTS}

\begin{abstract}
Abstrat
This century has been significantly marked by populational aging. In Vale do Taquari, Brazil, the number of elderly inhabitant already surpasses the national average, characterizing over 14\% of total population, according to the 2010 Census. The Social and Health Actions in Gerontology of Univates works with the elderly public of this region since 2014. This work's objective was to evaluate the social impact of said project, along with the participants in the year of 2016. The Elders answered about their satisfactions related to the return of the health evaluations ministered, the themes and information provided during the workshops and in relation to the impact of the project in their lives. The response average show that the elders considered the actions good and useful.

Keywords: Aging. University Extension. Social Impact. Elderly.

\section{EL IMPACTO DEL PROYECTO ACCIONES SOCIALES Y DE SALUD EN GERONTOLOGÍA/ UNIVATES EN LA POBLACIÓN ANCIANA}

\section{Resumen}

El siglo XXI viene siendo significativamente marcado por el envejecimiento poblacional. En el Vale do Taquari, Brazil, el número total de habitantes ancianos ya supera el promedio nacional, caracterizando el 14\% de la población total, según el Censo de 2010. El Proyecto Acciones Sociales y de Salud en Gerontología, de Univates, trabaja con el público de esta región desde 2014. El objetivo de este trabajo fue evaluar el impacto social del proyecto junto a los participantes en el año 2016. Los ancianos contestaron sobre sus satisfacciones con relación al retorno de las evaluaciones de salud hechas, a los temas trabajados e informaciones prestadas a lo largo de los talleres y en cuanto al impacto del proyecto en sus vidas. La media de las respuestas apunta que los ancianos juzgaron las acciones óptimas.

Palavras claves: Envejecimiento.Extensión Universitaria. Impacto Social. Ancianos. 
O impacto do projeto ações sociais e de saúde em Gerontologia/Univates (RS) para idosos

\section{INTRODUÇÃO}

O século XXI é caracterizado pela longevidade populacional, sendo que até 2014 a população idosa já representava 810 milhões de habitantes no contexto mundial, ou seja, $12 \%$ da população total (BODSTEIN; LIMA; BARRO, 2014). A Organização Mundial da Saúde estima que, até 2025, o Brasil seja considerado o sexto país do mundo em termos de população idosa. Para o ano de 2060, o órgão prevê que o percentual de idosos aumente para 30\% da população total.

Além disso, o censo demográfico de 2010 salienta que o Rio Grande do Sul (RS) passa por um período de intensas modificações, havendo um acréscimo no percentual de adultos e idosos e um decréscimo da população jovem. Tal fato tem grande relação com o crescimento da expectativa de vida, devido às melhores condições de saúde: o estado é o quarto colocado em relação à expectativa de vida dentre os estados da nação (BRASIL, 2010). No ano de 2014, o RS já era classificado como estado com maior proporção de população idosa: de cada seis pessoas, uma tinha 60 anos ou mais (IBGE, 2015) e, das 20 cidades com maior população de idosos, 13 localizam-se no RS (BRASIL, 2010).

No Vale do Taquari, o número de idosos também vem crescendo, e o percentual já ultrapassa a média nacional, que é de 8,6\%. A região abrange três dos cinco municípios brasileiros com o maior percentual de idosos: na primeira colocação, Coqueiro Baixo (29,4\%); na segunda, Relvado (26\%); na terceira, Colinas (25,4\%). Além disso, outros três municípios se destacam no país pela alta concentração de idosos entre seus habitantes: Imigrante $(24,18 \%)$, em $9^{\circ}$ lugar; Travesseiro (24,07\%), em $10^{\circ}$ lugar; e Forquetinha (23,2\%), em $12^{\circ}$ lugar. Até o ano de 2010, o Vale do Taquari já contava com 14\% de indivíduos acima de 60 anos, em sua população total (IBGE, 2011; IBGE, 2017).

Dentro desse contexto, percebeu-se a necessidade de desenvolver um projeto de extensão universitária que auxiliasse a Região do Vale do Taquari no desenvolvimento de políticas públicas visando à qualidade de vida da população idosa. A falta de dados que permitisse abrir essa discussão e de maior esclarecimento sobre como está acontecendo o envelhecimento justificou o planejamento e diretrizes do Projeto de Ações Sociais e de Saúde em Gerontologia(PASSG).

Freitas e Kopiller (2006) apontam o envelhecimento como um processo dinâmico e progressivo, no qual o ser humano passa por modificações morfológicas, funcionais, bioquímicas e psicológicas que geram a progressiva perda da capacidade de adaptação do indivíduo ao meio ambiente e o tornam suscetível a processos patológicos que terminam por levá-lo à morte.

Algumas dessas mudanças são de fácil visualização: perda da força e do vigor físico, visão curta, crescimento de pelos nas orelhas e narinas, problemas na memória de curto prazo, queda 
de cabelo, perda da massa óssea, diminuição da altura, diminuição da audição e menopausa. Existe uma variedade de mudanças associadas à idade que podem ser acrescentadas à essa lista. Essas transformações podem ser percebidas visualmente; suas causas, no entanto, não estão aparentes e são estabelecidas pelas inúmeras mudanças em todos os órgãos e tecidos, em cada célula que os compõe, incluindo-se aqui o sistema imunológico, endócrino e cardiovascular (HAYFLICK, 1996).

É importante frisar que essas transformações são gerais, e têm relação direta com a idade, com as características genéticas e, principalmente, com o modo de vida de cada um. A alimentação adequada, a prática de exercícios físicos, a exposição moderada ao sol, a estimulação mental, o controle do estresse, o apoio psicológico, a atitude positiva perante a vida e o envelhecimento são alguns fatores que podem retardar ou minimizar os efeitos da passagem do tempo (ZIMERMAN, 2000).

Nesse sentido, buscamos compreender o envelhecimento humano como um processo natural caracterizado por mudanças ocasionadas por uma multiplicidade de aspectos, os quais implicam modificações do interagir do indivíduo com o meio no qual está inserido. Conhecer a multidimensionalidade do processo do envelhecimento ao longo da vida é essencial tanto para compreender suas causas, como também para avaliar a necessidade de procedimentos para intervir, retardar e qualificá-lo (CORTELLETI; CASARA, 2007).

Acreditamos que a Universidade tem um papel não só educacional, como também social. Deve, pois, contribuir para a educação não-formal, a qual engloba a aquisição de conhecimentos e o desenvolvimento de competências e atitudes que permitam assumir uma vida ativa, autônoma e vivê-la de forma plena. O PASSG realizou avaliações de saúde, oficinas e palestras para os idosos participantes de grupos de convivência desta região. É essencial fomentar o desenvolvimento de medidas para garantir inclusão, acessibilidade e qualidade de vida a este público. No ano de 2016, 131 idosos participaram do projeto. Ao término das atividades, houve a entrega e explicação dos resultados das avaliações a cada idoso, em seu município. Constitui o objetivo deste artigo avaliar o impacto do PASSG para os idosos participantes.

\section{MATERIAIS E MÉTODOS}

O presente estudo caracteriza-se como quantitativo do tipo descritivo, de corte transversal. Foi desenvolvido na Universidade do Vale do Taquari - UNIVATES, localizada no município de Lajeado, na Região do Vale do Taquari, Rio Grande do Sul, Brasil. Participaram 7 (sete) municípios desta região, através de convite realizado às prefeituras. Após o aceite, mediante 
O impacto do projeto ações sociais e de saúde em Gerontologia/Univates (RS) para idosos

cartas de anuência dos municípios, iniciaram-se as atividades, em abril de 2016. Foram realizadas diversas avaliações com os seguintes instrumentos e protocolos avaliativos: Whoqol Bref, Peak Flow, Manovacuômetro, Timed up go (TUG), Dinamômetro, Relação Cintura Quadril (RCQ), IMC, Fototipo e Fotoenvelhecimento Cutâneo e Mini Exame do Estado Mental (MEEM). Após as avaliações e entregas de relatórios individuais aos idosos, foram realizadas oficinas de educação para um envelhecimento saudável, focalizando três temas que detectamos como fundamentais para estimular a mudança de hábitos de vida: exercícios respiratórios, risco de quedas e estimulação da memória.

Ao término das atividades, foi aplicado um instrumento avaliativo, no formato de questionário de impacto social das ações do PASSG, o qual teve a participação espontânea de 111 idosos. Os dados foram analisados em percentuais, a fim de quantificar suas percepções. O estudo ocorreu entre os meses de abril e dezembro de 2016.

\section{RESULTADOS E ANÁLISES}

O estudo abrangeu 131 idosos, sendo que 80,92\% ( $n=106)$ eram do sexo feminino e 19,08\% ( $\mathrm{n}=25)$ do sexo masculino. A idade média dos avaliados foi de 66 e 68 para o sexo feminino e masculino, respectivamente. Conforme a classificação de IMC segundo a Organização Pan-americana de Saúde (OPAS, 2002), dentre os avaliados 34,11\% ( $\mathrm{n}=44$ ) eram obesos; 32,56\% $(\mathrm{n}=42)$ apresentavam eutrofia; 22,48\% $(\mathrm{n}=29)$, sobrepeso; e 10,85\% $(\mathrm{n}=14)$, apresentaram baixo peso. Dois dos idosos não foram avaliados quanto a este quesito, devido ao não comparecimento na data de avaliação. Em relação ao estado civil dos participantes, 61,83\% (n=81) eram casados, 30,53\% ( $\mathrm{n}=40)$ viúvos, 5,34\% $(\mathrm{n}=7)$ solteiros e 2,29\% $(\mathrm{n}=3)$ separados. Dentre estes, 75,57\% $(\mathrm{n}=99)$ eram aposentados, 18,32\% $(\mathrm{n}=24)$ aposentados e pensionistas, $0,76 \%(\mathrm{n}=1)$ apenas pensionista, 2,29\% $(n=3)$ pensionistas ativos, 2,29\% $(n=3)$ remunerados ativos e $0,76 \%(n=1)$ não remunerado ativo.

As percepções avaliativas dos participantes mostraram-se positivas em relação ao impacto das ações realizadas, sendo que 96,5\% $(\mathrm{n}=86)$ afirmaram ter sido ótimo. Achados semelhantes foram encontrados em uma pesquisa junto a um Projeto de Extensão, com idosos da Faculdade de Enfermagem e Medicina, Nova Esperança (FACENE/FAMENE), de João Pessoa, Paraíba, na qual os participantes avaliaram as ações como de extrema importância para a vitalidade, autoestima e criação de vínculos. A média de idade dos avaliados nesse estudo era semelhante à da população do estudo em questão (GOMES et al., 2014 ). 
A respeito das informações técnicas prestadas durante as oficinas, $77,8 \%(\mathrm{n}=70)$ referiram ter sido ótimas. Tratando-se dos temas das oficinas ofertadas, $79,9 \%(\mathrm{n}=72)$ avaliaram como ótimas também. De forma semelhante, estudos de projetos de extensão universitária com idosos dos municípios de Rosana, Hortolândia e Sorocaba/SP e João Pessoa/PB, também demonstraram a satisfação dos idosos, constatando que estes sentem-se motivados e interessados pelas atividades desenvolvidas junto com eles. As oficinas de maior interesse relacionam-se à prática de atividade física, alimentação saudável, e inserção de novas tecnologias (HOPKA, 2014; GOMES et al., 2014; SOUZA; BARROS; VIANA, 2013; MARQUES et al., 2015).

Avaliando as atividades do PASSG como um todo, 86,58\% $(n=78)$ dos participantes julgaram-nas como ótimas e $78,81 \%(\mathrm{n}=71)$ ponderam o impacto do referido projeto em suas vidas, como ótimo de igual forma. Tais achados se assemelham à pesquisa de Gomes et al. (2014), em que os idosos referiram a importância do projeto de extensão em suas vidas.

As respostas positivas nos fazem refletir sobre os tipos de atividades que estão sendo desenvolvidas para os idosos nos municípios. Em pesquisa universitária em Florianópolis/SC, apontou-se a relevância de atividades físicas e de lazer proporcionadas pela instituição, sendo que esta possui evidências de oportunizar uma boa percepção de qualidade de vida dos idosos (SANTOS et al., 2014). Os idosos participantes do PASSG também possuem uma boa percepção de qualidade de vida e sentiram-se mais valorizados em poder estar próximos da universidade. Fundamentamos práticas que esclareceram dúvidas relativas ao envelhecimento, oportunizandolhes novas aprendizagens relativas a hábitos de vida a serem adotados para melhorar as condições de saúde. Esse público necessita de explicações sobre o processo de envelhecer e os cuidados necessários a serem tomados em seu dia a dia. Tais cautelas incluem desde suas limitações para não se colocarem em risco de quedas, ressaltando também a adoção de um estilo de vida ativo para o alívio de dores corporais e o controle de patologias. Instrumentalizar o idoso com conhecimentos e vivências pode ser fundamental para que ele envelheça com autonomia e de forma saudável.

Destacamos outro estudo realizado por Araújo et al. (2011), que analisou programas de promoção da saúde para idosos no Brasil. O estudo apontou a necessidade de proporcionar o empoderamento, isto é, tornar o idoso protagonista e atuante nas escolhas que determinam seu estado de saúde e inserção na sua teia social. Ele deve ser concretizado pela criação de um ambiente de suporte para a prevenção de agravos, aumento da capacidade funcional e melhoria da qualidade de vida, o que permite ampliar o conceito de saúde. Congruente com as ações realizadas no PASSG e as percepções dos idosos, aponta-se o impacto positivo destas na vida dos 
O impacto do projeto ações sociais e de saúde em Gerontologia/Univates (RS) para idosos

idosos participantes e evidencia-se a necessidade de continuar realizando atividades junto a este público.

\section{CONSIDERAÇÕES FINAIS}

Este estudo permitiu constatar a importância de realizar trabalhos com esta população, garantindo acesso à informação e cuidados em saúde, objetivando a melhora da qualidade e expectativa de vida. Consideramos fundamental o papel social da Universidade em fomentar as políticas públicas para os idosos, além de impactar positivamente na comunidade regional.

\section{REFERÊNCIAS}

ARAÚJO, L.F. et al. Evidências da contribuição dos programas de assistência ao idoso na promoção do envelhecimento saudável no Brasil. Rev Panam Salud Publica. 2011:30(1):80-6. Disponível em: <http://www.scielosp.org/pdf/rpsp/v30n1/v30n1a12.pdf $>$. Acesso em: 07.11.2017.

BODSTEIN, A.; LIMA, V.V.A; BARROS, A.M.A. A vulnerabilidade do idoso em situações de desastres: necessidade de uma política de resiliência eficaz. Rev. Amb. e Soc. São Paulo, v. 17, n. 2, p. 157-174, abr.-jun. 2014. Disponível em: $<$ http://www.scielo.br/scielo.php?script=sci_arttext\&pid=S1414-

753X2014000200011>. Acesso em:

06.11.2017.

BRASIL. Instituto Brasileiro de Geografia e Estatística. Indicadores Sociais Municipais: Uma Análise dos Resultados do Universo do Censo Demográfico 2010. 1. ed. Rio de Janeiro, RJ, 2011.

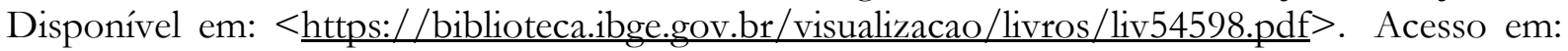
06.11.2017.

BRASIL. Instituto Brasileiro de Geografia e Estatística (IBGE). Síntese de indicadores sociais: uma análise das condições de vida da população brasileira. Rio de Janeiro, 2010. Disponível em: < http://biblioteca.ibge.gov.br/visualizacao/livros/liv45700.pdf $>$. Acesso em: 06.11.2017.

BRASIL. Instituto Brasileiro de Geografia e Estatística. Sistema IBGE de Recuperação Automática - SIDRA. São Paulo, 2017. Disponível em:

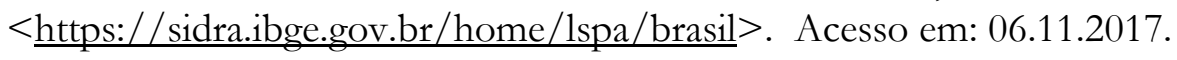

CORTELLETTI, Ivone A.; CASARA, Miriam B. Universidade da Terceira Idade - UNTI. $\begin{array}{lllll}\text { Caxias } & \text { do } & \text { Sul, } & \text { RS: }\end{array}$

FREITAS, E.V., KOPILLER, D. Atividade Física no Idoso. In: FREITAS, E.V. et al. Tratado de Geriatria e Gerontologia. 2.ed. Rio de Janeiro: Guanabara Koogan, 2006.

GOMES, H. M. et al. Extensão Universitária: a arte de cuidar de idosos. Rev. Ciênc. Saúde Nova Esperança, v.12, n.1, p.22-33, 2014. Disponível em: <http://www.facene.com.br/wpcontent/uploads/2010/11/EXTENS\%C3\%83O-UNIVERSIT\%C3\%81RIA-A-ARTE-DE- 
O impacto do projeto ações sociais e de saúde em Gerontologia/Univates (RS) para idosos

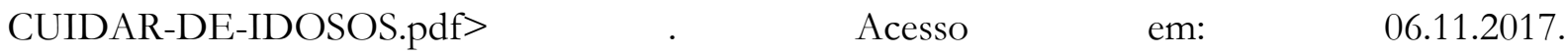

HAYFLICK, L. Como e por que envelhecermos. 2. ed. Rio de Janeiro: Campus, 1996. 366p.

HOPKA, F.R. O Projeto de Extensão Unati como ferramenta de inclusão de pessoas da terceira idade do município de Rosana/SP. 2014. 55 f. Monografia (Especialização) - Curso de Pós Graduação em Gestão Pública, Universidade Tecnológica Federal do Paraná (UTFPR), Pato Branco, 2014. Disponível em: $<$ http://repositorio.roca.utfpr.edu.br/jspui/bitstream/1/7016/1/PB GP IV 2014 04.pdf $>$.

Acesso em:

07.11 .2017

IBGE - Instituto Brasileiro de Geografia e estatística. Síntese de indicadores sociais: uma análise das condições de vida da população brasileira. Rio de Janeiro, 2015. Disponível em:

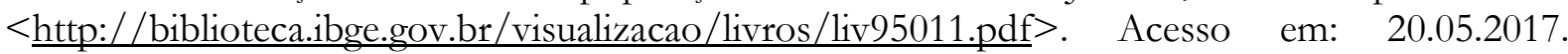

MARQUES, M. A. et al. Projeto de extensão universitária para idosos: inclusão digital e informática educativa. In: $8^{\circ}$ CONGRESSO DE EXTENSÃO UNIVERSITÁRIA DA UNESP, 2015, São Paulo. Artigos. São Paulo, 2015. Disponível em: $<$ https://repositorio.unesp.br/handle/11449/142068>. Acesso em: 08.11.2017.

SANTOS, P. M. dos et al. Atividades no lazer e qualidade de vida de idosos de um programa de extensão universitária em Florianópolis (SC). Rev Bras Ativ Fis Saúde v.19 n.4, p. 494-503, 2014. Disponível em:

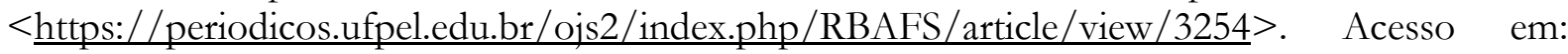
06.11 .2017$.

SOUZA, A.M. de, BARROS, M.J.A de, VIANA, H.B. Projeto de extensão universitária: possibilidades para a prática de atividades físicas e promoção da qualidade de vida em idosos. Rev. Dig. Buenos Aires, año 18, n. 183, 2013. Disponível em: $<$ http://www.efdeportes.com/efd183/promocao-da-qualidade-de-vida-em-idosos.htm $>$. Acesso em: 08.11.2017.

ZIMERMAN, Guite I. VELHICE - Aspectos Biopsicossociais. Porto Alegre: ARTMED, 2000 . 